\title{
Observation of narrow states in nuclei beyond the proton drip line: ${ }^{15} \mathrm{~F}$ and ${ }^{16} \mathrm{Ne}$
}

I. Mukha, ${ }^{1,2}$ N. K. Timofeyuk, ${ }^{3}$ K. Sümmerer, ${ }^{4}$ L. Acosta, ${ }^{5}$ M. A. G. Alvarez, ${ }^{1}$ E. Casarejos, ${ }^{6}$ A. Chatillon, ${ }^{4}$ D. Cortina-Gil,${ }^{6}$ J. M. Espino, ${ }^{1}$ A. Fomichev, ${ }^{7}$ J. E. García-Ramos, ${ }^{5}$ H. Geissel, ${ }^{4}$ J. Gómez-Camacho, ${ }^{1}$ L. Grigorenko, ${ }^{4,7}$ J. Hofmann ${ }^{4}$ O. Kiselev, ${ }^{4,8}$ A. Korsheninnikov, ${ }^{2}$ N. Kurz, ${ }^{4}$ Yu. Litvinov, ${ }^{4,9}$ I. Martel, ${ }^{5}$ C. Nociforo, ${ }^{4}$ W. Ott, ${ }^{4}$ M. Pfützner, ${ }^{10}$ C. Rodríguez-Tajes, ${ }^{6}$ E. Roeckl, ${ }^{4}$ M. Stanoiu, ${ }^{4,11}$ H. Weick, ${ }^{4}$ and P. J. Woods ${ }^{12}$

${ }^{1}$ Universidad de Sevilla, E-41012 Seville, Spain

${ }^{2}$ RRC "Kurchatov Institute," RU-123184 Moscow, Russia

${ }^{3}$ Department of Physics, School of Electronics Physical Sciences, University of Surrey, Guildford, GU2 7XH, United Kingdom

${ }^{4}$ GSI Helmholtzzentrum für Schwerionenforschung, D-64291 Darmstadt, Germany

${ }^{5}$ Universidad de Huelva, E-21071 Huelva, Spain

${ }^{6}$ Universidade de Santiago de Compostela, E-15782 Santiago de Compostela, Spain

${ }^{7}$ Joint Institute for Nuclear Research, RU-141980 Dubna, Russia

${ }^{8}$ Paul Scherrer Institut, CH-5232 Villigen, Switzerland

${ }^{9}$ Max-Planck-Institut für Kernphysik, D-69117 Heidelberg, Germany

${ }^{10}$ IEP, Warsaw University, PL-00681 Warszawa, Poland

${ }^{11}$ IFIN-HH, P. O. Box MG-6, Bucharest, Romania

${ }^{12}$ University of Edinburgh, EH1 1HT Edinburgh, United Kingdom

(Received 15 April 2009; revised manuscript received 11 June 2009; published 29 June 2009)

\begin{abstract}
Two high-lying states in ${ }^{15} \mathrm{~F}$ and ${ }^{16} \mathrm{Ne}$, unbound with respect to one-proton $(1 p)$ and two-proton $(2 p)$ emissions, have been observed in the fragmentation of ${ }^{17} \mathrm{Ne}$ at intermediate energies. They undergo mainly sequential emissions of protons via intermediate states in ${ }^{14} \mathrm{O}$ and ${ }^{15} \mathrm{~F}$ and have decay energies of 7.8(2) and 7.6(2) $\mathrm{MeV}$, respectively. The widths of the newly observed states in ${ }^{15} \mathrm{~F}$ and ${ }^{16} \mathrm{Ne}$ are much smaller than the Wigner limits for single-particle configurations, of $0.4(4)$ and $0.8\left({ }_{+8}^{-4}\right) \mathrm{MeV}$, respectively. In addition, narrow widths of $0.2(2) \mathrm{MeV}$ are derived for two other high-lying states in ${ }^{15} \mathrm{~F}$ with $Q_{p}$ of 4.9 and $6.4 \mathrm{MeV}$, which match features of the recently predicted narrow odd-parity ${ }^{15} \mathrm{~F}$ states with two valence protons in the $s d$ shell. All energies and widths have been obtained by analyzing angular correlations of the decay products, $p-p-{ }^{14} \mathrm{O}$ and $p-p-{ }^{13} \mathrm{~N}$, whose trajectories have been measured by a tracking technique with silicon microstrip detectors.
\end{abstract}

DOI: 10.1103/PhysRevC.79.061301

PACS number(s): 21.10.-k, 21.45.-v, 23.50.+z, 27.20.+n

Nuclear structure beyond the proton drip line, where nuclei exist only as resonances in the continuum, remains rather unexplored. In light nuclei, such resonances are usually expected to be very broad because of the small Coulomb barriers. Thus, the ground states (g.s.) of ${ }^{15} \mathrm{~F}$ or ${ }^{10,11} \mathrm{~N}$ are seen as broad $s$-wave proton resonances. In contrast, $1 p$ emitters in heavy nuclei live much longer because of the much higher Coulomb barriers. Unexpectedly long half-lives have been also reported for the $2 p$ emitters ${ }^{45} \mathrm{Fe},{ }^{54} \mathrm{Zn},{ }^{19} \mathrm{Mg}$, and ${ }^{94 m} \mathrm{Ag}$ [1-4]. A quantum-mechanical theory of the $2 p$ radioactivity based on a three-body model [5] explains them as a result of the considerable influence of few-body centrifugal and Coulomb barriers together with nuclear structure effects. It predicts the regular occurrence of long-lived $2 p$ precursors.

Recently, Canton et al. [6] suggested that some unbound states could exist as very narrow resonances. Using multichannel algebraic scattering (MCAS) theory, Canton et al. predicted three odd-parity states in ${ }^{15} \mathrm{~F}$, namely, $\frac{1}{2}^{-}, \frac{5}{2}^{-}$, and $\frac{3}{2}^{-}$, with the widths of only a few $\mathrm{keV}$. These predictions were challenged by Fortune and Sherr [7], who argued that the MCAS results contradicted both the mirror symmetry and the $(s d)^{2}$ shell-model systematics.

There are two important aspects of the predictions made by the MCAS and shell model. (i) The ${ }^{15} \mathrm{~F}$ odd-parity states predicted by both Refs. [6] and [7] lie in the vicinity of the $2 p$ threshold. Therefore, three-body ${ }^{13} \mathrm{~N}+p+p$ dynamics, which cannot be taken into account by either the shell model or the MCAS, may strongly influence their properties. The predicted states lie somewhere around the $1 p$ thresholds ${ }^{14} \mathrm{O}\left(1^{-}\right)+p,{ }^{14} \mathrm{O}\left(0_{2}^{+}\right)+p,{ }^{14} \mathrm{O}\left(3^{-}\right)+p$ etc. Therefore, additional $2 p$ and $1 p$ decay channels may be open. For structural reasons, the partial widths of these channels may be larger than those from the ${ }^{14} \mathrm{O}_{\text {g.s. }}+p$ channel. Thus, the odd-parity ${ }^{15} \mathrm{~F}$ states may not be as narrow as predicted in Refs. [6,7]. Such a phenomenon may be general for nuclei beyond the proton drip line, where $1 p$ and $2 p$ thresholds are very low. (ii) Accurate predictions of resonance positions and widths are crucial in studies of stellar nucleosynthesis. The shell model, widely used for these purposes, is incapable of generating continuum wave functions. It only provides spectroscopic factors $\left(C^{2} S\right.$ determined from occupancies of bound orbits), which are multiplied then by single-particle widths $\Gamma_{\text {s.p. }}$ calculated elsewhere. In contrast, the MCAS provides continuum wave functions, and some authors even claim that it is able to deal with the shell melting phenomenon by introducing Pauli hindrance in its scheme. However, its validity has not yet been tested.

In our previous work [8], we reported two new resonances in ${ }^{15} \mathrm{~F}$ populated by $1 p$ decay of ${ }^{16} \mathrm{Ne}$. In this paper, we estimate their widths by analyzing the data taken in addition to the study 


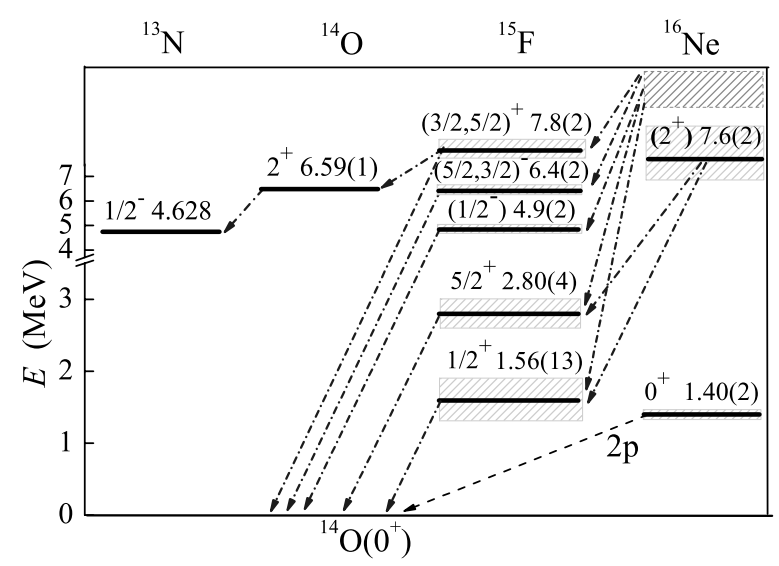

FIG. 1. $1 p$ decays (dash-dot arrows) of states in ${ }^{16} \mathrm{Ne},{ }^{15} \mathrm{~F}$, and ${ }^{14} \mathrm{O}$. The $2 p$ decay of ${ }^{16} \mathrm{Ne}$ (g.s.) is shown by a dashed arrow. The hatched area indicates unspecified states in ${ }^{16} \mathrm{Ne}$.

of ${ }^{19} \mathrm{Mg}$ and ${ }^{16} \mathrm{Ne}$ (g.s.) $[3,8]$. We also observe new narrow excited states in the decay chain ${ }^{16} \mathrm{Ne}^{*} \rightarrow{ }^{15} \mathrm{~F}^{*}+p \rightarrow{ }^{14} \mathrm{O}^{*}+$ $p+p \rightarrow{ }^{13} \mathrm{~N}+p+p+p$ and investigate their properties shown in Fig. 1.

The experiment was performed with a $591 \mathrm{~A} \mathrm{MeV}$ beam of ${ }^{24} \mathrm{Mg}$ accelerated by the SIS facility at GSI, Darmstadt. The radioactive beam of ${ }^{17} \mathrm{Ne}$ was produced at the projectilefragment separator FRS [9] with an intensity of 800 ions $\mathrm{s}^{-1}$ and an energy of $450 \mathrm{~A} \mathrm{MeV}$. The secondary reactions $\left({ }^{17} \mathrm{Ne},{ }^{16} \mathrm{Ne}^{*}\right)$ occurred at the midplane of FRS in a secondary ${ }^{9} \mathrm{Be}$ target. The first half of FRS was adjusted to transmit ${ }^{17} \mathrm{Ne}$ ions, and its second half was tuned for identification of the residual heavy ions (HI), e.g., ${ }^{14} \mathrm{O}$ and ${ }^{13} \mathrm{~N}$. A microstrip detector array, developed on the basis of the AMS02 particle tracker [10], was positioned downstream of the secondary target. It consisted of four silicon microstrip detectors with a strip pitch of $100 \mu \mathrm{m}$ covering an opening angle of $\sim 150 \mathrm{mrad}$ around the secondary beam direction. The arrangement of the detectors can be found in Ref. [3]. They were used to measure energy loss and positions of each particle in triplecoincidence events $\mathrm{HI}+2 p$, thus allowing a reconstruction of trajectories of all decay products, the coordinates of the reaction vertex, and the angular $p$-HI correlations. The achieved transverse position accuracy was $30 \mu \mathrm{m}$ for protons and $15 \mu \mathrm{m}$ for ${ }^{14} \mathrm{O}\left({ }^{13} \mathrm{~N}\right)$. The angular resolution gained in tracking of fragments was $\sim 1 \mathrm{mrad}$. More details concerning the detector performance and the tracking procedure are given in Refs. $[3,8]$.

The ${ }^{15} \mathrm{~F}^{*}$ and ${ }^{16} \mathrm{Ne}^{*}$ states were identified from the measured $\mathrm{HI}+2 p$ events and their decay energies were derived by analyzing angular correlations between the protons and the ${ }^{13} \mathrm{~N}\left({ }^{14} \mathrm{O}\right)$ ions. Such a procedure is similar to an identification of a reaction channel by using a Dalitz plot, which is illustrated in Fig. 2(a). Two protons from the same parent state share the $2 p$-decay energy $Q_{2 p}$, thus their momenta are located along the arc area with a constant root-sum-squared momentum. Two $2 p$-decay mechanisms can be distinguished in Dalitz plots: (i) sequential emission of protons, which may be described as two consecutive $1 p$ decays with two $k_{p \text {-HI }}$ peaks reflecting the respective $p$-HI resonances, and (ii) simultaneously emitted
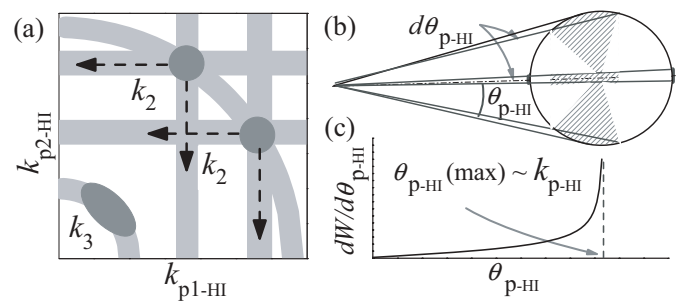

FIG. 2. (a) Transverse momentum correlations $k_{p 1-\mathrm{HI}}-k_{p 2-\mathrm{HI}}$ for a direct three-body $\left(k_{3}\right)$ and sequential $\left(k_{2}\right) 2 p$-decay mechanisms. Arrows show directions of the peak tails. (b) Kinematical enhancement of angular $p$-HI correlations at the maximum possible angle for a given $k_{p-\mathrm{HI}}$. (c) The corresponding angular $p$-HI distribution.

protons with continuous $p$-HI spectra peaked around $Q_{2 p} / 2$ [11]. In Fig. 2(a), these mechanisms are shown in the respective kinematical areas $k_{2}$ and $k_{3}$. A single $2 p$-parent state yields two peaks along the corresponding arc area. Several such states decaying through the same intermediate $1 p$ resonance reveal "slices", as shown in Fig. 2(a), reflecting $p$-HI final state interactions (FSI) due to resonances in the corresponding states. The angular $\theta_{p 1-\mathrm{HI}}-\theta_{p 2-\mathrm{HI}}$ correlations show similar structures. Because of a strong kinematical focusing at intermediate energies, $1 p$ decay leads to a sharp angular $p$-HI correlation, see Figs. 2(b) and 2(c). The $p$-HI angles reflect the transverse proton momentum relative to HI, and they are correlated with the precursor's decay energy. Thus, sequential $2 p$ decays result mostly in the peaks located along the arc areas in the angular $\theta_{p 1-\mathrm{HI}}-\theta_{p 2-\mathrm{HI}}$ correlations, similar to those sketched in Fig. 2(a).

The angular $\theta_{p 1-\mathrm{O}}-\theta_{p 2-\mathrm{O}}$ correlations derived from the measured ${ }^{14} \mathrm{O}+p+p$ events and their projections on $\theta_{p-\mathrm{O}}$ are shown in Fig. 3. Most events are seen at larger angles and originate from the $2 p$ decay of excited states in ${ }^{16} \mathrm{Ne}$. However, the events with smaller angles cluster around $\theta_{p-\mathrm{O}}=35 \mathrm{mrad}$. They are attributed to the $2 p$ decay from ${ }^{16} \mathrm{Ne}_{\text {g.s. }}$ [8]. These events were disentangled by making a slice projection from the measured correlations with the gate $\theta_{p 2-\mathrm{O}}<45 \mathrm{mrad}$ [peak (1) in Fig. 3(b)]. The data were compared to a Monte Carlo simulation of the response of our setup to a direct $2 p$ decay ${ }^{16} \mathrm{Ne} \rightarrow{ }^{14} \mathrm{O}+p+p$ with the known $Q_{2 p}$ energy by using the GEANT software [12]. The calculations took into account the experimental uncertainties of tracking the fragments when reconstructing the vertex coordinates and the angles of fragment trajectories. The simulation reproduced the data quantitatively with $Q_{2 p}=$ $1.35(8) \mathrm{MeV}$, in agreement with the literature value of 1.4(1) $\mathrm{MeV}$ [13].

Figure 3(c) displays the $\theta_{p 1-0}$ distribution obtained by gating on $\theta_{p 2-\mathrm{O}}>120 \mathrm{mrad}$, which corresponds to FSI in $p-{ }^{14} \mathrm{O}$ pairs due to the low-lying states in ${ }^{15} \mathrm{~F}$. The simulations of $1 p$ decays of the known ground $\frac{1}{2}^{+}$and first-excited $\frac{5}{2}^{+}$states in ${ }^{15} \mathrm{~F}$ with $Q_{p}$ of $1.5(1)$ and $2.80(5) \mathrm{MeV}$ [14], respectively, reproduced the two smallest angle peaks quantitatively [8]. The two peaks at larger angles were assigned to $1 p$ decays of unknown excited states in ${ }^{15} \mathrm{~F}$ with derived $Q_{p}$ values of 4.9(2) and 6.4(2) MeV. These values and their uncertainties were deduced similarly to those inferred for 

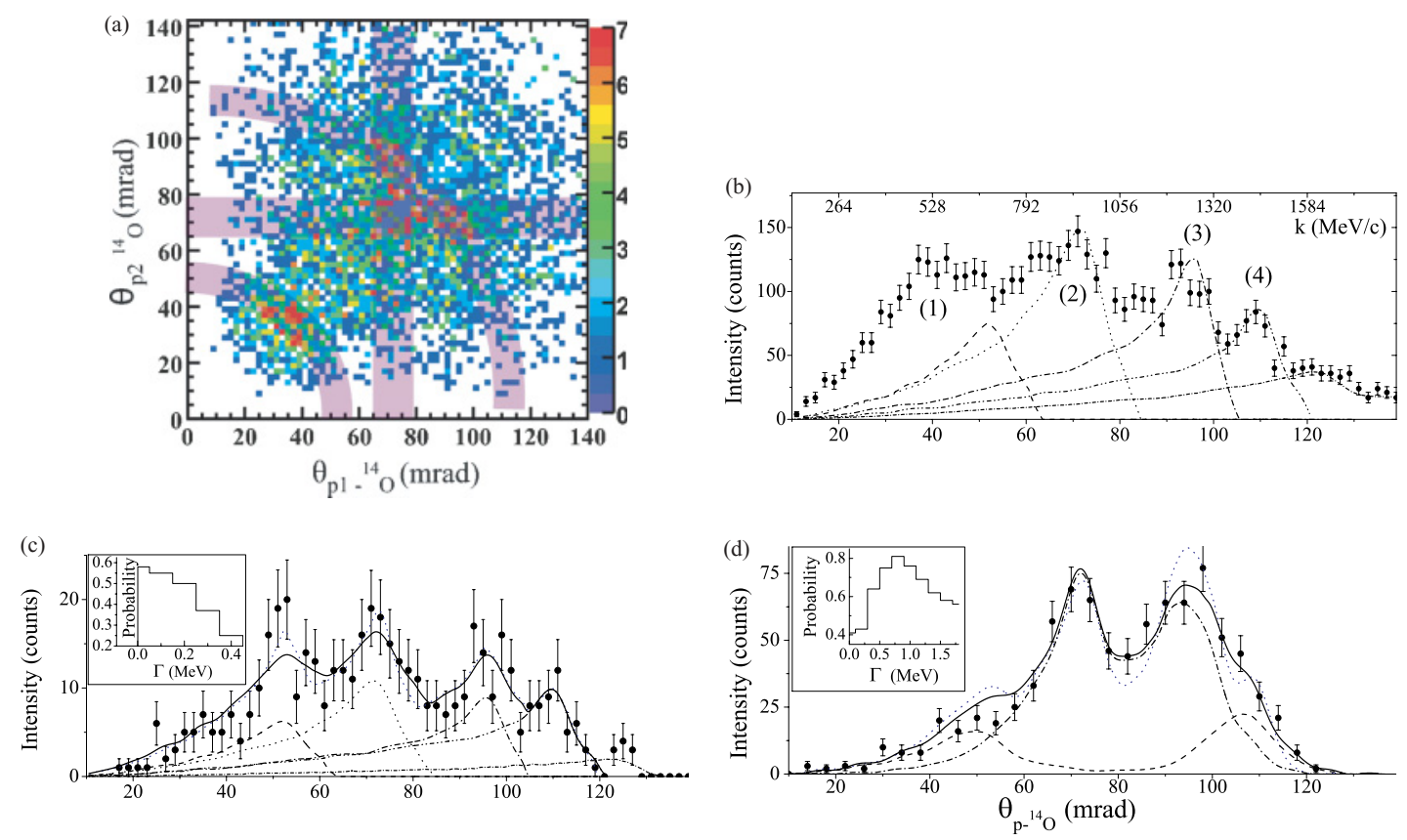

FIG. 3. (Color online) (a) Angular $\theta_{p_{1}-\mathrm{O}^{-}} \theta_{p_{2}-\mathrm{O}}$ correlations obtained from the measured ${ }^{14} \mathrm{O}+p+p$ events. The lilac areas indicate

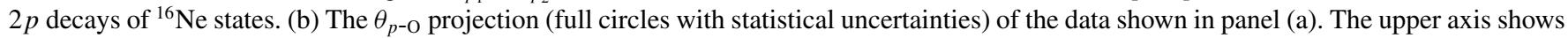
the transverse momenta $k$ of protons with respect to ${ }^{14} \mathrm{O}$. The apparent peaks are labeled (1)-(4). The curves are similar to those in (c). (c) The $\theta_{p_{1} \text {-o }}$ distribution obtained by gating on $\theta_{p_{2} \text {-O }}>120 \mathrm{mrad}$, which corresponds to ${ }^{15} \mathrm{~F}$ resonances due to $p_{1^{-}}{ }^{14} \mathrm{O}$ FSI. The dash and dot curves are the simulations of the setup response to the known $1 p$ decays of the ground and first-excited states in ${ }^{15} \mathrm{~F}$, see Fig. 1 . The dash-dot and dash-dot-dot curves indicate two new states in ${ }^{15} \mathrm{~F}$ with fitted $Q_{p}$ values of $4.9(2)$ and $6.4(2) \mathrm{MeV}$, respectively. The solid line is the sum fit. The short-dash curve shows the sum fit with all level widths set to $1 \mathrm{keV}$. The short-dash-dot curve is the $1 p$-decay estimate of the $7.8 \mathrm{MeV}$ state in ${ }^{15} \mathrm{~F}$. (d) The $\theta_{p \text {-O }}$ distribution selected within the arc-area $\sqrt{\theta_{p 1-\mathrm{O}}^{2}+\theta_{p 2-\mathrm{O}}^{2}}$ around $115 \mathrm{mrad}$, which corresponds to the $7.6 \mathrm{MeV}$ state in ${ }^{16} \mathrm{Ne}$. The solid curve is a fit obtained by simulating the sequential $2 p$ decay of the ${ }^{16} \mathrm{Ne}^{*}$ state via the ground (dash curve) and the first-excited (dash-dot curve) state in ${ }^{15} \mathrm{~F}$. The dot curve shows a similar fit with the ${ }^{16} \mathrm{Ne}^{*}$ width set to $1 \mathrm{keV}$. The inset shows the probability (as a function of the assumed resonance width) that the simulations match the data.

${ }^{16} \mathrm{Ne}_{\text {g.s. }}$ [8]. Namely, for a chosen range of $Q_{p}$ we calculated the probability $P\left(Q_{p}\right)$ for simulations to match the data (the standard statistical Kolmogorov test [15]). The $Q_{p}$ value with the closest match (assuming that $P>50 \%$ ) was accepted, and its uncertainty was taken as the half-width of the distribution where $P \geqslant 50 \%$. The resonance widths $\Gamma$ were fitted similarly. The $\frac{1}{2}^{+}$and $\frac{5}{2}^{+}$states in ${ }^{15} \mathrm{~F}$ with known widths served as test cases. The 4.9 and $6.4 \mathrm{MeV}$ levels are very narrow, the conservative estimate of both widths is $0.2(2) \mathrm{MeV}$ [e.g., see the $4.9 \mathrm{MeV}$ width fit in the inset of Fig. 3(c)]. These values were taken as upper-limits reflecting the resolution of the setup.

The 4.9 MeV state is likely the mirror state of ${ }^{15} \mathrm{C}\left(\frac{1}{2}^{-}\right)$ since its location relative to the $\frac{5}{2}_{1}^{+}$state is similar to that in ${ }^{15} \mathrm{C}$. This state is $\sim 0.3 \mathrm{MeV}$ higher than the $\frac{1}{2}^{-}$state calculated in the $(s d)^{2}$ shell model but is $0.6 \mathrm{MeV}$ lower than the MCAS prediction, being just $0.27 \mathrm{MeV}$ above the $2 p$ threshold. The $2 p$-decay branch is strongly suppressed, and the ${ }^{14} \mathrm{O}\left(0_{1}^{+}\right)+p$ decay mode dominates. The data do not allow us to distinguish between the shell-model or MCAS predictions for its width. The $6.4 \mathrm{MeV}$ state in ${ }^{15} \mathrm{~F}$ is also open for $2 p$ decay and may be seen in triple ${ }^{13} \mathrm{~N}+p+p$ coincidences. The angular $\theta_{p_{1}-\mathrm{N}}-\theta_{p_{2}-\mathrm{N}}$ correlations obtained from the triple events are shown in Fig. 4(a). Only a few events are detected in the arc area of interest around $62 \mathrm{mrad}$, so these data are not conclusive. The $6.4 \mathrm{MeV}$ state is open to sequential $2 p$ decays via the $1_{1}^{-}, 0_{2}^{+}, 3_{1}^{-}$states of ${ }^{14} \mathrm{O}$. To estimate the widths $\Gamma=C^{2} S \times \Gamma_{\text {s.p. }}$ of these unobserved decays, we calculated $\Gamma_{\text {s.p. }}$ in the two-body potential model with the Woods-Saxon potential parameters $r_{0}=1.25 \mathrm{fm}$ and $a=$ $0.65 \mathrm{fm}$, and we calculated $C^{2} S$ in the spsdpf-shell model with the WBP interaction [16] using the NUSHELL@MSU code [17]. Two possible $J^{\pi}$ assignments for the $6.4 \mathrm{MeV}$ state were considered, $\frac{5}{2}^{-}$and $\frac{3}{2}^{-}$. In both cases, the dominant structure is a $d$-wave proton outside the ${ }^{14} \mathrm{O}\left(1^{-}\right)$core. However, because of the centrifugal barrier, the $\Gamma_{\text {s.p. }}$ for this configuration is only $\sim 11 \mathrm{keV}$, while the same proton is well above the barrier for the ${ }^{14} \mathrm{O}\left(0_{1}^{+}\right)+p$ branch with $\ell=1$. Although $C^{2} S$ for the $\ell=1$ branch is small, the ${ }^{14} \mathrm{O}\left(0_{1}^{+}\right)+p$ width dominates, being $\sim 50 \mathrm{keV}$ for $\frac{5}{2}^{-}$and more than $100 \mathrm{keV}$ for $\frac{3}{2}^{-}$. The corresponding widths for the ${ }^{14} \mathrm{O}\left(1^{-}\right)+p$ decay are about 4 and $7 \mathrm{keV}$. This explains our nonobservation of the $2 p$ decay of the $6.4 \mathrm{MeV}$ state. Although both $J^{\pi}$ assignments predict relatively narrow widths consistent with the data, the energy split between the 4.9 and $6.4 \mathrm{MeV}$ states favors the $\frac{3}{2}^{-}$ assignment, since the same split between the $\frac{1}{2}^{-}$and $\frac{3}{2}^{-}$states 

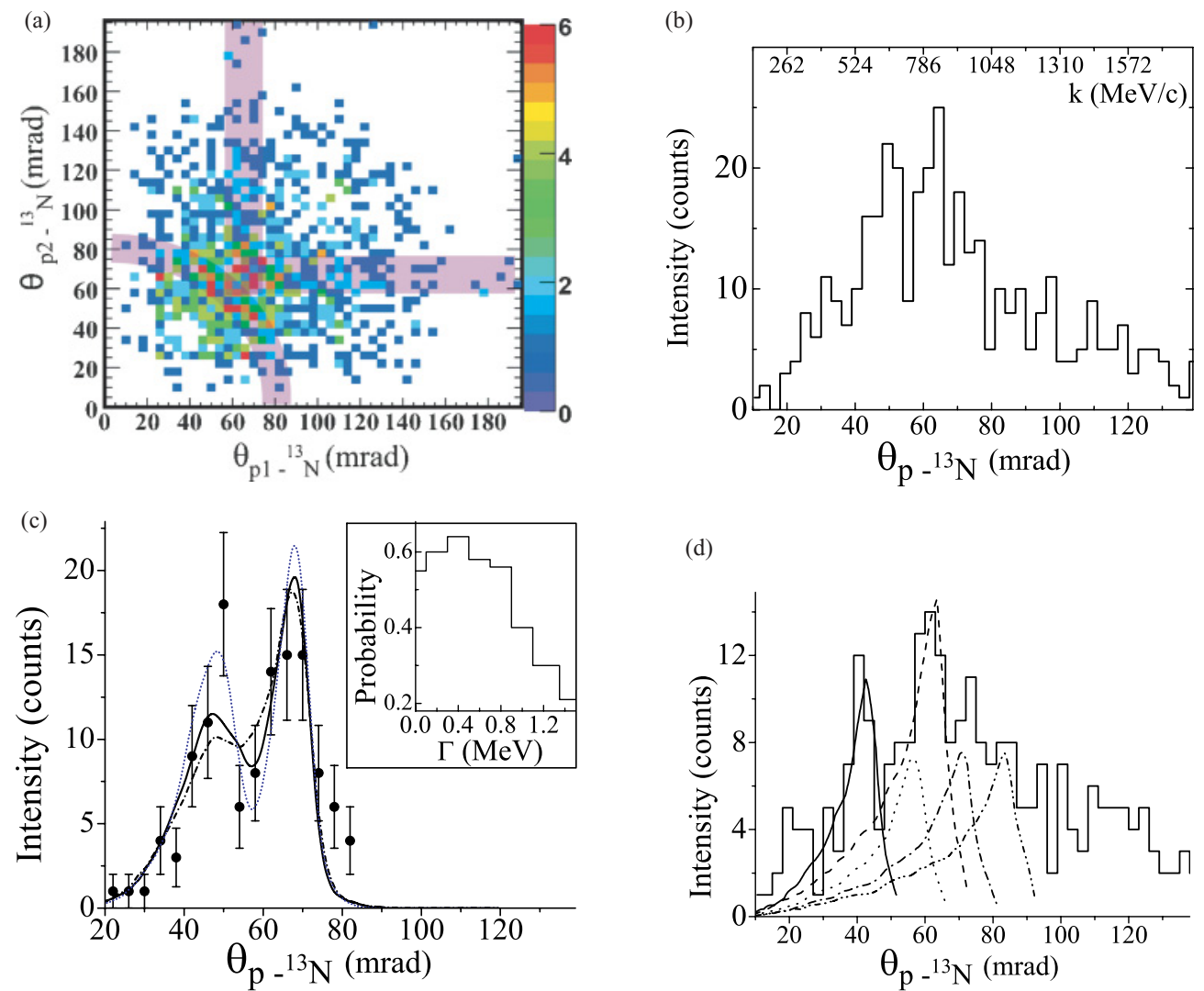

FIG. 4. (Color online) (a) Angular $\theta_{p 1-\mathrm{N}}-\theta_{p 2-\mathrm{N}}$ correlations from the ${ }^{13} \mathrm{~N}+p+p$ events. The arc area indicates $2 p$ emission from an unknown ${ }^{15} \mathrm{~F}^{*}$ resonance. The bands show the $p+{ }^{13} \mathrm{~N}$ FSI due to the $3^{-}$state in ${ }^{14} \mathrm{O}$. (b) Projection $\theta_{p-\mathrm{N}}$ histogram of the data shown in panel (a). (c) The $\theta_{p-\mathrm{N}}$ distribution (full circles with statistical errors) gated on $78 \leqslant \sqrt{\theta_{p 1-\mathrm{N}}^{2}+\theta_{p 2-\mathrm{N}}^{2}} \leq 88 \mathrm{mrad}$, which corresponds to the $2 p$ decay of a resonance in ${ }^{15} \mathrm{~F}^{*}$. The solid curve is the simulation of the sequential $2 p$ decay of ${ }^{15} \mathrm{~F}^{*}$ via the $2^{+}$state in ${ }^{14} \mathrm{O}$ at $E^{*}=6.59 \mathrm{MeV}[18]$. The fitted parameters of the ${ }^{15} \mathrm{~F}^{*}$ state are $Q_{p}=7.8(2) \mathrm{MeV}$ and $\Gamma=0.4(4) \mathrm{MeV}$. The dot and dash-dot curves show similar calculations with assumed ${ }^{15} \mathrm{~F}^{*}$ widths of 1 and $800 \mathrm{keV}$, respectively. The inset shows the probability (as a function of the assumed resonance width) that the simulations match the data. (d) The $\theta_{p 1-\mathrm{N}}$ histogram obtained for $\theta_{p 2-\mathrm{N}}>80 \mathrm{mrad}$, which corresponds to FSI in $p+{ }^{13} \mathrm{~N}$. The solid, dot, dash, dash-dot, and dash-dot-dot curves are simulations of the $1 p$ resonances in ${ }^{14} \mathrm{O}^{*}$ at $E^{*}$ of $5173,5920,6272,6590$, and $7768 \mathrm{keV}$, respectively [18].

is observed in the mirror nucleus ${ }^{15} \mathrm{C}$. The $\frac{3}{2}^{-}$assignment also agrees with the shell-model predictions [7] (see Table I).

The triple ${ }^{13} \mathrm{~N}+p+p$ coincidence indicates the presence of a new state in ${ }^{15} \mathrm{~F}$. Two intense bumps are seen around 50 and $65 \mathrm{mrad}$ in the $\theta_{p-\mathrm{N}}$ projection without any gate
[Fig. 4(b)]. We selected these peaks by the arc gate of 78$88 \mathrm{mrad}$ [Fig. 4(c)]. In the corresponding $\theta_{p-\mathrm{N}}$ distribution, two distinguished peaks have positions and widths matching those from the sequential $2 p$ decay of a narrow ${ }^{15} \mathrm{~F}^{*}$ level via the known state ${ }^{14} \mathrm{O}\left(2_{1}^{+}\right)$at $6.59 \mathrm{MeV}[18]$. This is justified

TABLE I. $Q_{p}^{\exp }$ and $\Gamma_{p}^{\exp }$ (in MeV) of states observed in ${ }^{15} \mathrm{~F}^{*}$, the assigned spin-parity $J^{\pi}$, the calculated spsdpf shell-model widths $\Gamma_{p}^{\mathrm{SM}}$ in comparison with the $(s d)^{2}$ shell model [6] and the MCAS [7] predictions. The excitation energies $E_{x}^{15} \mathrm{C}$ of ${ }^{15} \mathrm{C}$ mirror states are from Ref. [18].

\begin{tabular}{lcccccccc}
\hline \hline$Q_{p}^{\exp }$ & $\Gamma_{p}^{\exp }$ & $J^{\pi}$ & $\Gamma_{p}^{\mathrm{SM}}$ & $Q_{p}^{[6]}$ & $\Gamma_{p}^{[6]}$ & $Q_{p}^{[7]}$ & $\Gamma_{p}^{[7]}$ & $E_{x}^{15 \mathrm{C}}$ \\
\hline $2.80(5)$ & $0.4(1)$ & $5 / 2^{+}$ & 0.33 & 2.78 & 0.3 & 2.79 & 0.18 & 0.74 \\
$4.9(2)$ & $0.2(2)$ & $1 / 2^{-}$ & 0.09 & 5.49 & 0.005 & 4.63 & 0.055 & 3.10 \\
$6.4(2)$ & $0.2(2)$ & $5 / 2^{-}$ & 0.05 & 6.88 & 0.010 & 5.92 & 0.006 & 4.22 \\
& & $3 / 2^{-}$ & 0.10 & 7.25 & 0.040 & 6.30 & 0.180 & 4.66 \\
$7.8(2)$ & $0.4(4)$ & $3 / 2_{2}^{+}$ & $0.45^{\mathrm{a}}$ & - & - & - & - & 5.83 \\
& & $1 / 2_{2}^{-}$ & $\sim 3^{\mathrm{b}}$ & - & - & - & - & 5.87 \\
& $5 / 2_{2}^{+}$ & $0.3^{\mathrm{a}}$ & 7.75 & 0.4 & - & - & 6.36 \\
\hline \hline
\end{tabular}

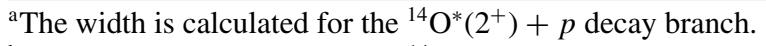

${ }^{\mathrm{b}}$ The width is calculated for the ${ }^{14} \mathrm{O}^{*}\left(1^{-}\right)+p$ decay branch. 
by the FSI channel ${ }^{13} \mathrm{~N}+p \rightarrow{ }^{14} \mathrm{O}^{*}$ whose $\theta_{p-\mathrm{N}}$ correlations are shown in Fig. 4(d). The simulations of the known 5.17 and $6.59 \mathrm{MeV}$ states in ${ }^{14} \mathrm{O}[18]$ match the two most intense peaks of the distribution. The fitted $Q_{2 p}$ value for the ${ }^{15} \mathrm{~F}^{*}$ state is $3.2(2) \mathrm{MeV}$ and $\Gamma=0.4(4) \mathrm{MeV}$. The derived width is actually an upper-limit estimate, see the inset of Fig. 4(c). The new ${ }^{15} \mathrm{~F}^{*}$ state is also open to the ${ }^{14} \mathrm{O}\left(0_{1}^{+}\right)+p$ decay by $Q_{p}=7.8(2) \mathrm{MeV}$. We have simulated this channel using the ${ }^{15} \mathrm{~F}^{*}$ energy and width derived from the observed $2 p$ branch, see Fig. 3(b). Some data events may be attributed to the $1 p$ decay, though contributions from other possible higher-lying states in ${ }^{15} \mathrm{~F}$ are unknown. Thus we estimate the ratio of the $1 p / 2 p$ decay branches of the $7.8 \mathrm{MeV}$ state to be less than 0.2 . Three $J^{\pi}$ assignments were considered for this state, $\frac{3}{2}{ }_{2}^{+}, \frac{1}{2}_{2}^{-}$, and $\frac{5}{2}_{2}^{+}$, based on known spin-parities in the mirror nucleus ${ }^{15} \mathrm{C}$. The spsdpf shell-model widths of the main decay channels for each of these assignments are given in Table I. The $\frac{1}{2}_{2}{ }^{-}$assignment is clearly wrong. We cannot discriminate between the $\frac{3}{2}_{2}^{+}$and $\frac{5}{2}_{2}^{+}$assignments by using the measured $1 p / 2 p$ branching ratio, because the $1 p$ decay width cannot be reliably determined in our theoretical approach. However, this energy matches well the MCAS predictions for $\frac{5}{2}_{2}^{+}$.

Searching for reaction channels feeding the observed ${ }^{15} \mathrm{~F}$ states, we inspected two bumps in Fig. 3(a) around the $p-{ }^{14} \mathrm{O}$ angles of 70 and $100 \mathrm{mrad}$. These bumps were assumed to originate from sequential $2 p$ decay of a single excited state in ${ }^{16} \mathrm{Ne}$ via ${ }^{15} \mathrm{~F}$. The $\theta_{p-\mathrm{O}}$ distribution, selected within the corresponding arc area and shown in Fig. 3(d), can be explained by sequential $2 p$ decay of a high-lying ${ }^{16} \mathrm{Ne}^{*}$ state via ${ }^{15} \mathrm{~F}\left(\frac{1}{2}^{+}\right)$and ${ }^{15} \mathrm{~F}\left(\frac{5}{2}^{+}\right)$with the fitted values $Q_{2 p}=$ 7.6(2) $\mathrm{MeV}$ and $\Gamma_{p}=0.8\left(\begin{array}{c}-4 \\ +8\end{array}\right) \mathrm{MeV}$. The $P(\Gamma)$ distribution for this state is shown in the inset of Fig. 3(d). The asymmetric shape of $P(\Gamma)$ is due to correlation of two fit parameters, the level width and the decay branching ratio, when larger assumed widths cause smaller admixtures of the $\frac{1}{2}^{+}$decay channel. The obtained branching ratios of the $\frac{1}{2}^{+}$and $\frac{5}{2}^{+}$decay channels are $0.24(8)$ and $0.76(8)$, respectively. The position of the observed ${ }^{16} \mathrm{Ne}^{*}$ state corresponds to the $6.1 \mathrm{MeV}$ state in its mirror ${ }^{16} \mathrm{C}$ with $J^{\pi}=\left(2^{+}, 3^{-}, 4^{+}\right)$[13]. We have calculated
TABLE II. Different $J^{\pi}$ assignment for the $7.6 \mathrm{MeV}$ level in ${ }^{16} \mathrm{Ne}$ and the corresponding partial widths (in $\mathrm{MeV}$ ) for decay into three ${ }^{15} \mathrm{~F}+p$ channels, calculated in the shell model.

\begin{tabular}{lccc}
\hline \hline$J^{\pi}$ & ${ }^{15} \mathrm{~F}\left(\frac{1}{2}_{1}^{+}\right)+p$ & ${ }^{15} \mathrm{~F}\left(\frac{5}{2}_{1}^{+}\right)+p$ & ${ }^{15} \mathrm{~F}\left(\frac{1}{2}^{-}\right)+p$ \\
\hline $2^{+}$ & 0.036 & $>0.37$ & 0.036 \\
$3^{-}$ & 0.007 & $>0.005$ & 0.120 \\
$4^{+}$ & & 1.4 & \\
\hline \hline
\end{tabular}

shell-model partial widths of all decay channels for each of these $J^{\pi}$ assignments to the observed ${ }^{16} \mathrm{Ne} *$ state. The most important ones of them are shown in Table II. According to these calculations, the only plausible spin-parity of the 7.6 $\mathrm{MeV}$ state is $2^{+}$.

Our nuclear-state assignments assume that one peak in the measured $p$-HI spectra matches one single resonance only. Therefore several closely spaced states could be misinterpreted as one broad level if they are populated within the experimental resolution of $0.2-0.4 \mathrm{MeV}$. Simulations of the response of our setup show that multiple scattering of the protons in the thick target is the main reason for the $p$-HI peak broadening and the relatively large errors of the resonance width measurements. The ${ }^{15} \mathrm{~F}$ and ${ }^{16} \mathrm{Ne}$ data could be improved significantly in new experiments with a thinner target.

All in all, the measured $1 p$ and $2 p$ decays of the new states in ${ }^{15} \mathrm{~F}$ and ${ }^{16} \mathrm{Ne}$ give evidence for relatively stable nuclear configurations beyond the proton drip line. The observed states have much smaller widths than those expected for protons moving around undisturbed nuclear cores. Their structure may be understood as protons orbiting excited cores which are in turn open to $1 p$ decays. Such a phenomenon challenges the current nuclear structure theories, which cannot yet accommodate the three-body character of the $2 p$ decays into the many-body nature of atomic nuclei.

We thank M. Pohl, E. Cortina, and DPNC coworkers from the Université de Génève for developing the microstrip detectors and acknowledge support of the EURONS EC-I3, Spanish FPA2006-13807-C02-01, and UK STFC ST/F012012/1 grants.
[1] M. Pfützner et al., Eur. Phys. J. A 14, 279 (2002); J. Giovinazzo et al., Phys. Rev. Lett. 89, 102501 (2002).

[2] B. Blank et al., Phys. Rev. Lett. 94, 232501 (2005).

[3] I. Mukha et al., Phys. Rev. Lett. 99, 182501 (2007).

[4] I. Mukha et al., Nature (London) 439, 298 (2006).

[5] L. V. Grigorenko, R. C. Johnson, I. G. Mukha, I. J. Thompson, and M. V. Zhukov, Phys. Rev. Lett. 85, 22 (2000).

[6] L. Canton, G. Pisent, J. P. Svenne, K. Amos, and S. Karataglidis, Phys. Rev. Lett. 96, 072502 (2006); 99, 089202 (2007).

[7] H. T. Fortune and R. Sherr, Phys. Rev. Lett. 99, 089201 (2007); H. T. Fortune, Phys. Rev. C 74, 054310 (2006).

[8] I. Mukha et al., Phys. Rev. C 77, 061303(R) (2008).

[9] H. Geissel et al., Nucl. Instrum. Methods Phys. Res. B 70, 286 (1992).
[10] B. Alpat et al., Nucl. Instrum. Methods Phys. Res. A 540, 121 (2005).

[11] L. V. Grigorenko, I. G. Mukha, I. J. Thompson, and M. V. Zhukov, Phys. Rev. Lett. 88, 042502 (2002).

[12] GEANT-detector simulation tool, CERN software library, http://wwwasd.web.cern.ch/wwwasd/geant.

[13] D. R. Tilley et al., Nucl. Phys. A564, 1 (1993).

[14] A. Lepine-Szily et al., Nucl. Phys. A734, 331 (2004).

[15] W. T. Eadie et al., Statistical Methods in Experimental Physics (North-Holland, Amsterdam, 1971).

[16] B. A. Brown, Prog. Part. Nucl. Phys. 47, 517 (2001).

[17] B. A. Brown and W. D. M. Rae, NUSHELL@MSU, MSU-NSCL Report, 2007 (unpublished).

[18] F. Ajzenberg-Selove et al., Nucl. Phys. A523, 1 (1991). 